\title{
Towards understanding of artificial intelligence in accounting profession
}

\section{Dr Yajnya Dutta Nayak}

Assistant Professor

Pg Dept. Of Commerce

Khallikote Auto. College, Berhampur

India

Mr. Aditya Prasad Sahoo

Phd Research Scholar

Ksom, Kiit University, Bhubaneswar

Email: adityasahoo007@gmail.com

India

\section{A R T I C L E I N F O}

Article history: Article

Received: $\quad 25$ April 2021

Revised: $\quad 20$ May 2021

Accepted: $\quad 28$ May 2021

DOI: $10.47742 / i j b s s r . v 2 n 5 p 1$

\section{A B S T R A C T}

Accountants have embraced the emission of automation over many years to get better the efficiency and effectiveness of their work. But technology has not been able to replace the need for expert knowledge and decision-making. Earlier generations of 'intelligent systems have usually demonstrated the progressing power of human expertise and the restrictions of machines. In the upcoming decades, intelligent systems must take over more and better decision-making tasks from humans. While accountant has been using technology for a lot of years to improve what they do and deliver more value to businesses, this is an opportunity to reimagine and radically improve the quality of business and investment decisions which is the ultimate purpose of the profession. Accountants, as expert decision-makers, use both ways of thinking they apply their knowledge to specific situations to make reasoned decisions, although also make quick intuitive decisions based on extensive experience in their field. Today, Al is being used for image recognition, object identification, detection, classification, and automated geophysical feature detection. These are underlying tasks that once required the input of a human. Focusing on how artificial intelligence will impact accountants, Al will very soon help the organization to automate much of the routine and repetitive activities that are undertaken on a daily, weekly or annual basis. It will also help the organization to empower quick decision-making to create smart insights examine huge quantities of data with ease.

Keywords: Artificial Intelligence (AI), Knowledge-based Systems \& Accounting Profession

\section{Introduction}

Accountants have embraced the emission of automation over many years to get better the efficiency and effectiveness of their work. But technology has not been able to replace the need for expert knowledge and decision-making. earlier generations of 'intelligent systems have usually demonstrated the progressing power of human expertise and the restrictions of machines. Artificial intelligence (AI) has been an aspiration of computer scientists since the 1950s, and it has seen tremendous progress in recent years. Examples of AI are already an integral part of many of our online activities and will become increasingly embedded in everything it touches and does. These systems do not replicate human intelligence. Indeed, several experts would dispute the exercise of the term 'AI' at all to describe recent machine learning systems. Though, on a task-by-task starting point, systems increasingly produce outputs that far go beyond the accuracy and consistency of those produced by humans.
In the short to medium term, Artificial intelligence (AI) brings a lot of opportunities for accountants to improve their efficiency, provide more insight and deliver more value to businesses. In the longer term, Artificial intelligence (AI) raises opportunities for much more radical change, as systems increasingly take over decision-making tasks currently done by humans.

\section{History of artificial intelligence}

Advances in computer hardware and computer software in the ' 60 s and ' 70 s have led to increased use of computers by people in all walks of life. The scientists and researchers involved in the design and development of these physical devices have relentlessly worked to find various applications for these machines. The idea of having intelligent assistants to human beings that were haunting many logicians, philosophers, novelists, and futurists for quite a long time started getting crystallized with the availability of these machines. For example, 
Vol: 2, Issue: 5

May/2021

https://ijbssrnet.com/index.php/ijbssr

DOI: http://dx.doi.org/10.47742/ijbssr.v2n5p1

http://iarpnet.org/

George Boole (1815to1864), who invented Boolean algebra, in the preface of his book titled an Investigation of The Laws Of Thought On Which Are Founded The Mathematical Theories Of Logic And Probabilities (1854) writes: "The laws we have to examine are the laws of one of the most important of our mental faculties. The mathematics these have to construct is the mathematics of the human intellect". This reveals the fact that his concern was more in the direction of intelligent machines or artificial intelligence rather than mathematics alone. Perhaps the best citation that one can exert is that of Alan Turing who perspicaciously felt the possibility of computer intelligence. Turing, in his very widely accepted article Computing Machinery and Intelligence have explicitly expressed that a computer could be programmed to exhibit intelligent behavior. Such thoughtful speculations have resulted in the ascendancy of a new area of research called Artificial Intelligence (A.I.).

The 1950s started the "real" development of Artificial intelligence $(\mathrm{AI})$ and related programming languages. During the early 1950s, Arthur Samuel of IBM developed the first gameplaying program for checkers. The Samuel machine learning programs were responsible for the high performance of the checkers game player. By the madisons, the term "Artificial Intelligence" was coined by John McCarthy of MIT at the 1956 Dartmouth Conference, the first conference devoted to AI. Later in 1958, he invented the LISP language. At almost the same time, Allen Newell, J.C. Shaw, and Herbert Simon wrote and demonstrated the Logic Theorist (LT), the first running Artificial intelligence (AI) program. The trio later demonstrated a General Problem Solver program. The decade ended with Marvin Minsky and John McCarthy starting a laboratory at MIT devoted to the study of Artificial intelligence (AI).

\section{Law and Knowledge-based Systems}

Artificial Intelligence deals with the development of cognitive models and computer programs to emulate the intelligence of human beings. In the initial early work in artificial intelligence, researchers and system developers attempted to develop general problem solvers. However, these efforts were met with several impediments. In the early '70s, Feigenbaum and associates suggested that artificial intelligence techniques could be made more effective by adding domain knowledge. At this point, it is worth citing the following statement of Feigenbaum made in: “.... general problem-solvers are too weak to be used as the basis for building high-performance systems. The behavior of the best problem-solvers these know, human problem-solvers, is observed to be weak and shallow, except in the areas in which the human problem-solver is a specialist. And it is observed that the transfer of expertise between specialty areas is slight. A chess master is dubious to be a specialist algebraist or a professional mass spectrum analyst, etc. In this view, the expert is the specialist, with a specialist's knowledge of his area and a specialist's methods and heuristics."

\section{Artificial Intelligence}

Authors defined Artificial Intelligence (AI) in different ways. For example, (Winston, 1984) has defined Artificial intelligence (AI) as "the study of ideas which enable computers to be intelligent." (Kurzweil, 1990) defined Artificial intelligence (AI) as "the art of creating machines that perform functions that require intelligence when performed by people". According to (Lugar; Stubblefield, 1993) Artificial intelligence (AI) is "the branch of computer science that is concerned with the automation of intelligent behavior".

Artificial intelligence is one of the computer science fields relating to improving the intelligence of computerization to emulation the human intelligence, and the expert systems one branch of the artificial intelligence, these systems implement duties popular implement by the especial and expert human. In the past decades, there have been numerous articles about the application of Artificial intelligence (AI) as "expert systems", which are called by some investigators "knowledge-based systems" also.

\section{Artificial intelligence technology}

Artificial intelligence systems can be awfully powerful and are improving quickly. They offer outputs that be able to extremely accurate, replacing and, in several cases, far superseding human efforts. However, they do not replicate human intelligence. It requires recognizing the strengths of this different form of intelligence, and build an understanding of the best traditions for humans and computers to effort together.

\section{Accountancy \& Artificial intelligence}

Although artificial intelligence techniques such as machine learning are not new, and the pace of change is fast, widespread adoption in business and accounting are still in their early stages. To build a positive vision of the future, that requires extending a deep understanding of how artificial intelligence can solve accounting and business problems, the practical challenges and the skills accountants require to work alongside intelligent systems.

\section{Artificial intelligence (AI) and the profession}

In the upcoming decades, intelligent systems must take over more and better decision-making tasks from humans. While accountant has been using technology for a lot of years to improve what they do and deliver more value to businesses, this is an opportunity to reimagine and radically improve the quality of business and investment decisions which is the ultimate purpose of the profession. Accountants want to help organizations and economies work better by giving good advice and making good decisions. After all, accounting is not an end in itself. All the activities associated with accounting ultimately aim to help people make good decisions about the allocation of resources, and hold others to account for their decisions. This underpins investment, growth, and confidence in all organizations and economies.

More intelligent systems enable radically different approaches to this ultimate objective and the kinds of fundamental business problems the profession aims to solve. Investors require to gain confidence and trust in the financial results of companies; companies and governments require to ensure the correct levels of tax are paid; management requires to decide how to spend organizational resources, and be held accountable for those decisions. Solving these kinds of 
Vol: 2, Issue: 5

May/2021

https://ijbssrnet.com/index.php/ijbssr

DOI: http://dx.doi.org/10.47742/ijbssr.v2n5p1

http://iarpnet.org/

fundamental problems is essential for companies and economies to succeed and is at the heart of the accountancy profession.

There may also be new problems that can be transformed with new data and more intelligent systems. As an example, the UN Global Goals represent a broad consensus of what we, as humans, want the world and society to look like in the coming decades. In all cases, there is an enormous amount of work to do to achieve those goals. At the very least, these require effective measurement to enable informed decisions on the allocation of financial and other resources to achieve the goals. These also require accountability for those decisions. The starting point for creating a longer-term vision is So to focus on the profession's ultimate purpose of better decisions and identify the fundamental business problems that better decisions will solve.

\section{Exploit Powerful Technologies}

It is then significant to recognize and exploit the power of innovative technologies effectively. This report highlights the strength of machine learning approaches to AI, and deep learning methods in particular, which are regularly leading to major advancements in a lot of areas of research. But it is a complex and ever-altering technological context. Other areas of technology will interact with Artificial intelligence (AI) and have a significant impact on business in the future, like blockchain or quantum computing. In addition, the pace of alter in capabilities be capable of very fast, and the nature of learning based and information-driven systems enable continual improvement.

To fully exploit powerful new technologies, these require to be clear about their unique characteristics and how they can help to solve real problems. There is characteristically a long time from the structure of working technology to getting the utmost value from it. Regularly, technology can be a solution looking for a difficulty to solve, or simply enables us to replicate how these do things using different tools. Instead, these require to encourage debate, interaction and learning between technology experts, business, and the profession to reimagine the way that solves fundamental business problems with the help of new technologies.

In doing this, the profession also requires to be open to more profound change and avoid just defending or incrementally improving the status quo. Where Artificial intelligence (AI) enables greater insight from data, it helps human experts make better decisions and provide better advice. Indeed, evidence suggests that humans and computers working together in areas as diverse as chess and medicine produce better results than humans or computers in isolation.

However, as systems continue to get more powerful, they will be able to move further into complex decision areas, potentially replacing humans altogether in a lot of cases and enabling entirely different solutions, services and models. When looking at the longer term, So, the profession must think beyond incremental improvements to existing processes. Furthermore, it requires reflecting on the specific skills and qualities that accountants bring to businesses.

This also means engaging positively in debates on, for example, the role of human judgment in more complex business areas. There may well be uniquely human characteristics, such as leadership, empathy, and creativity, which will never be replaced by computers. And it should not underestimate the adaptability and ingenuity of humans. However, 'human judgment' is often just a substitute for lack of data powerful computers with access to new sources of data may well supersede the requirement for human judgment in the vast majority of cases. Attempts to deny the potential of computers to surpass a lot of human capabilities, and simply to protect current models and ways of doing things, are likely to fail.

It is impossible to predict the extent to which computers will replace human decision-making over the next 20 to 30 years. There is much broader context and the long-term future of accountancy will ultimately reflect how this, as humans, sees and shape our relationship with powerful systems. This will be influenced by a range of economic, social, and political factors.

The technology of the future will also be very different from what businesses see today. As a result, a flexible approach is essential when thinking about the future. The skills and learning agenda for the future accounting professional, in particular, has been subject to a lot of debate between professional bodies, employers, and educators. Most would agree that accountants will require more hard skills in areas such as technology and data, as well as a greater emphasis on things like soft skills, critical thinking, and adaptability. There also requires to be greater emphasis on life-long learning. However, there are competing longer-term visions of the future accountant from highly qualified hybrid professionals to lower-skilled workers, who use technology to access expert knowledge. When business requires change, and all the relevant stakeholders agree, the profession can adapt. Professional bodies change their qualifications as market demands evolve. Accountancy organizations in business and practice innovate all the time to provide more value. This quality will become increasingly important for all stakeholders involved in the profession.

\section{Impact Artificial intelligence}

Accountants, as expert decision-makers, use both ways of thinking they apply their knowledge to specific situations to make reasoned decisions, although also make quick intuitive decisions based on extensive experience in their field.

Today, AI is being used for image recognition, object identification, detection, classification, and automated geophysical feature detection. These are underlying tasks that once required the input of a human. Focusing on how artificial intelligence will impact accountants, AI will very soon help the organization to automate much of the routine and repetitive activities that are undertaken on a daily, weekly or annual basis. It will also help the organization to: empower quick decision-making create smart insights examine huge quantities of data with ease.

A. Predictive and forecasting solutions: Helping an organization's clients forecast the finances of their business is an extremely valuable element offered by the organization's practice. With Artificial intelligence (AI) integrated into the software, the organization will be able to provide a comprehensive and accurate insight for the organization's clients 
Vol: 2, Issue: 5

May/2021

https://ijbssrnet.com/index.php/ijbssr

DOI: http://dx.doi.org/10.47742/ijbssr.v2n5p1

http://iarpnet.org/

without the usual "manual heavy lifting" and number crunching behind report creation. On a day-to-day basis, being able to quickly and easily access up-to-date and truthful reports and forecasts can help organizations form a closer and more useful relationship with the organization's clients.

B. Smart assistants: In an organization accountant who, during crunch time when seemingly every client is sending through their accounts, considers turning off the organization's contact no or email so the organization can get some work done. Fortunately, smart assistants might be able to provide the organization a helping hand.

C. Automatic tagging and allocation of transactions: The next two areas where AI will help an organization's accounting practice are also enabled by machine learning. This will save the organization time by correctly tagging transactions and assigning them to the right ledger account. Put simply, an organization's accounting software will learn from previous tagging decisions that are typically made according to rules that the accountant is aware of. Some of these rules are intuitive however others can be surprisingly complex, at least from a computer's point of view.

D. Anomaly detection: Computers love data, of course, and when machine learning is applied to massive amounts of data such as the yearly ledgers of a large company then there are clear benefits. The organization will be able to discover anomalies that may exist, and the process will be much quicker and take significantly less effort.

E. OCR solutions: Optical character recognition (OCR) isn't new although Artificial intelligence (AI) enhances its accuracy extensively and opens it to new usage scenarios. it has always been possible to take out information or data mechanically from documents, this required a human to point out to the OCR software where the data was situated something that as well meant the document layout could not be malformed with no further instruction. Computers have always known what numbers are, of course. That's what defines a computer. This means the salient data can be extracted to allow information or data to be allocated and/or processed by the software rather than by human action even if the software hasn't seen a receipt like that before, or if the scanned document isn't particularly high quality.

\section{Approaches to Artificial Intelligence}

This approach had some success although it rarely produced results that could be seen as akin to human intelligence. While there were a variety of technical issues with such systems, they were ultimately defeated by the complexity of the real world, and the extent to which rely on intuitive thinking. Organizations were unable to articulate our knowledge and decision-making rules enough. This supposed that systems could not manage with a composite or ambiguous situation, or where possessions changed.

Recent successes in Artificial intelligence (AI) take a very different approach. This uses pattern acknowledgment and is known as machine learning. While there are a lot of fields of research into Artificial intelligence (AI), improvements in machine learning are the main drivers behind the hype around Artificial intelligence (AI) today and the focus of this report. By combining approaches in machine learning with developments in other areas of AI, such as knowledge representation and reasoning, computers can be used to complement and increasingly improve both ways of human thinking.

\section{Accountants following Artificial intelligence (AI)}

Although Artificial intelligence (AI) techniques such as machine learning are not new, and the pace of change is fast, widespread adoption in business and accounting is still in its early stages. To build a positive vision of the future, organizations require to extend a deep understanding of how Artificial intelligence (AI) can solve accounting and business problems, the practical challenges and the skills accountants require to work alongside intelligent systems.

Accountants apply their technical knowledge about accounting and finance to help businesses and stakeholders make better decisions. To support their decision-making and advice, accountants require high-quality financial and non-financial information or data and analysis. This is reflected in a wide range of accountancy roles across business and practice to capture, prepare, check and communicate information or data, undertake analysis, and make a wide variety of decisions. Accountants have been deploying technology for a lot of years to help them provide better advice and make better decisions. Technology can help them do this by solving three broad problems:

- It is providing better and cheaper data to support better decision-making;

- It is automatically generating new insights from the data analysis; and

- It is freeing up time to focus on more expensive tasks such as good decision making, solving problems, managing advising, relationship building, strategy increase, and leadership.

So, it is important to identify accounting and business problems where machine learning is likely to be particularly fruitful and where problems may be less appropriate for these techniques. This will ensure that adoption efforts are driven by business requirements, rather than simply technology capabilities. To date, there has been limited use in real-world accounting although early research and implementation projects include:

- using machine learning to code accounting entries and improve on the accuracy of rules-based approaches, enabling greater automation of processes;

- improving fraud detection through additional sophisticated, machine learning models of normal' activities and better prediction of fraudulent activities;

- using machine learning-based predictive models to forecast revenues; and

- improving access to, and analysis of, unstructured data, such as contracts and emails, through deep learning models. 
Vol: 2, Issue: 5

May/2021

https://ijbssrnet.com/index.php/ijbssr

DOI: http://dx.doi.org/10.47742/ijbssr.v2n5p1

http://iarpnet.org/

\section{Challenges of Artificial Intelligence (AI)}

Data volumes and quality are crucial to the success of Artificial intelligence (AI) systems. Without sufficient good information or data, models will not be able to receive or learn. Transactional accounting data is well-structured and high quality, and thus should be a promising starting point for developing models. Powerful models may require external sources of data, which may not always be possible to access at an appropriate cost. So, building experience of both successful and less successful cases will help to inform future adoption.

Another additional principled limit will be privacy and ethics, especially where Artificial intelligence (AI) systems are drawing on personal data. Primary, machine learning is increasingly becoming integrated into business and accounting software. As a result, a lot of accountants will encounter machine learning without realizing it, similar to how organizations use these capabilities in their online searching or shopping activities. This is how smaller organizations in particular are most likely to adopt Artificial intelligence (AI) tools. Second, conscious adoption of Artificial intelligence (AI) capabilities to solve specific business or accounting problems will often require substantial investment. Given the data volumes involved, substantial hardware and processing power may be required, even if it is accessed on a cloud basis. As a result, Artificial intelligence (AI) investments will likely focus on areas that will have the biggest financial impact, especially cost reduction opportunities or those that are crucial for competitive positioning or customer service. Other areas, while potentially beneficial, may lack a strong investment case. Likewise, using machine learning to extend additional intelligent products in specialist accounting areas may lack the market potential to justify investment from software developers.

\section{Conclusion}

Accounting has a wider institutional context, and regulators and standard setters also require creating their thoughtful application of Artificial intelligence (AI) and being comfortable with any linked risks. Without this institutional support, it is very difficult to achieve change in the areas of finance or accounting. So, the active participation of standard setters and regulators in these areas is essential. For example, standard setters in the account will want to look at where accountants are using these techniques to gain evidence and understand the reliability of these techniques. Such bodies are already assessing the impact of data analytics capabilities on finance and accounting should build on those discussions.

\section{Reference}

Brynjolfsson, E. and McAfee, A. (2014), The Second Machine Age: Work, Progress, and Prosperity in a Time of Brilliant

Technologies, New York: W. W. Norton \& Company.

Government Office for Science, (2016), Artificial intelligence: opportunities and implications for the future of decision making,

Government Office for Science.

ICAEW, (2015), Providing Leadership in a Digital World, London: ICAEW.

ISCA and ICAEW, (2017), Our Future Together: Industry Perspectives on the Future of Professional Learning and Entrepreneurship, ISCA and ICAEW.

Kahneman, D. (2012), Thinking, Fast and Slow, London: Penguin. Kahneman, D. and Klein, G. (2009), 'Conditions for Intuitive Expertise: A Failure to Disagree', American Psychologist, Vol. 64, No. 6, pp.515-526.

McKinsey Global Institute, (2017), A Future that Works: Automation, Employment, and Productivity, San Francisco: McKinsey \& Company.

Nilsson, N. (2009), The Quest for Artificial Intelligence: A History of Ideas and Achievements, Cambridge: Cambridge University Press.

O’Neil, C. (2016), Weapons of Math Destruction: How Big Data Increases Inequality and Threatens Democracy, New York: Crow Publishing Group.

Russell, S. and Norvig, P. (2010), Artificial Intelligence: A Modern Approach, London: Pearson. Susskind, R. and Susskind, D. (2015), The Future of the Professions: How Technology Will Transform the Work of Human Experts, Oxford: Oxford University Press.

The Royal Society (2017), Machine learning: the power and promise of computers that learn by example, London: The Royal Society. 